$-203-$

ANALYSIS OF HOLTER MONITORING FOR THE ASSESSMENT OF VENTRICULAR PREMATURE CONTRACTIONS AND THE EVALUATION OF DRUG EFFICACY -ESTIMATION OF AN EFFICIENT METHOD OF ANALYSIS-

\author{
Yoshihiro Nishimura, Kenjiro Ishida, \\ Yasushi Motooka, Hiroaki Yoshida, \\ Shusuke Miwa, Yoshinori Asaoka, \\ Mutsumi Yoshida, Hideo Imamura \\ Nippon Steel Corporation Hirohata Works \\ Hospital
}

To establish the plain and correct method for assessing ventricular premature contractions (VPCs) using Holter monitoring and its clinical application for evaluating drug efficacy, consecutive two or three days Holter monitorings before and after administration of medicine were undertaken in 40 patients and the recordings were analysed using both manual method (M) and the automatic analyser $(\mathrm{Aa})$.

The calculated VPC frequencies obtained from one mimute and one hour monitorings, obserbed value multiplied by 1440 (VPC $1 \mathrm{~m} \times 1440$ ) and observed value multiplied by 24 (VPC $1 \mathrm{~h} \times 24$ ), respectively, were significantly different from the observed value by $M$ (VPC-M) $(p<0.001)$. VPC counts of Aa had a significant correlation with VPC-M $(r=0.820, p<0.001)$, but they were also significantly different from each other $(p<0.01)$. Sum total of the number of VPCs for one minute in every one hour multiplied by 60 ( $\Sigma$ VPC $1 \mathrm{~m} \times 60)$ correlated significantly with VPC-M $(\mathrm{r}=0.933$, $\mathrm{p}<0.001)$ and no difference was remarked between them. The $95 \%$ confidence intervals of variability between first and second day regarding VPC-M was $88 \%$ and the characteristics of VPCs also varied day by day. The minimal interval of VPC (VPC-MI) had a correlation with VPC-M $(r=0.574, p<0.001)$ and were mainly observed when the drug efficacy was considered to be lost in the medicated stage.

Conclusion 1) The most efficient method to evaluate VPCs is the combination of the physician's pattern analysis of the compression recordings of Holter monitorings at a glance and the use of $\Sigma$ VPC $1 \mathrm{~m} \times 60$ for VPC frequency. 2) Spontaneous day by day variations of VPCs are remarkable and a 24 hours monitoring is insufficient to evaluate the severity of VPCs and the efficacy of drugs. 3) VPC-MI might be an index for assessing drug efficacy.

\section{$-204-$ \\ A STUDY OF THE ACCELERATING EFFECT OF VERAPAMIL ON JUNCTIONAL AUTOMATICITY IN PATIENTS WITH SICK SINUS SYNDROME}

\footnotetext{
Shigeki Inaba, Hiroshi Kasanuki. Satoshi Ohnishi, Etsuko Tanaka, Jun Umemura, Takashi Nirei, Nobuhisa Magosaki, Kohshichiroh Hirosawa.

Department of Cardiology, The Heart Institute of Japan, Tokyo Women's Medical College.
}

In order to evaluate the accelerating effect of Verapamil (Ver) on junctional automaticity (JA), atrial overdrive suppression tests were performed before and after intravenous administration of $5 \mathrm{mg}$ of Ver in patients (pts) with SSS. Number of junctional beats and their recovery time (JRT) during 10 recovery cycles after pacing were measured for each pacing rate. In 15 pts junctional recovery beats were noticed both before and after administration of Ver, enabling comparison of Ver effect on JA. Acceleration of JA with Ver was defined as more than $10 \%$ shortening in JRT after administration of Ver. JA was accelerated in $10 \mathrm{pts}$. Among these, max JRT was shortened from $4256 \pm 1474$ to $2236 \pm 996 \mathrm{mSec}$, ratio of junctional beats among recovery beats increased from $50.2 \pm 22.2$ to $80.9 \pm 17.7 \%(\mathrm{p}<0.01)$ the pacing rate at which Wenkebach block appeared (W-rate) decreased from $152 \pm 24$ to $133 \pm 20 \mathrm{BPM}, \max$ JRT during pacing rates less than W-rate also shortened from $3542 \pm 1382$ to $2208 \pm 1018 \mathrm{mSec}$ $(\mathrm{p}<0.0 \mathrm{I})$ and systolic arterial pressure during $1: 1$ AV conduction pacing changed from $134 \pm 34$ to $122 \pm 17 \mathrm{mmHg}(\mathrm{n}=6$, N.S.). The 10 pts were divided into two groups according to the manner of acceleration of JA. After administration of Ver 7 pts showed lesser fluctuations in JRT $(<400 \mathrm{mSec})$ over different pacing rates [Group I] while the other 3 showed larger fluctuations $(>1500 \mathrm{mSec})$ [Group II]. Max JRT of group I and II were $1667 \pm 461$ and $3540 \pm 468$ $\mathrm{mSec}(\mathrm{p}<0.01)$, respectively. Lack of warming up of JA was noted in 3 of group I and none of group II. Organic heart disease was noted in 6 of group I and none of group II.

Thus Ver accelerated JA in $10 / 15$ pts with SSS. Although the mechanism of acceleration is unknown. suppression of AV node conduction and fall in arterial pressure with Ver did not seem to play a major role. And the two distinctive manner of JA acceleration noted in the 10 pts suggests involvement of at least two mechanisms.

\section{$-205-$ \\ EFFECT OF ADENOSINE-5'-TRIPHOSPHATE ON ATRIOVENTRICULAR REENTRANT TACHYCARDIA IN INFANCY AND CHILDHOOD}

Naoya Okumura, Akimasa Ogawa, Masaki Matsushima, Masami Nagashima*

Department of Pediatric Cardiology, Chukyo Hospital *Department of Pediatrics, Nagoya University School of Medicine

The effects of adenosine-5'-triphosphate (ATP) on atrioventricular reentrant tachycardia (AVRT) were studied in 6 patients aged 2 months to 6 years. There were 3 patients with WPWdynftomr, 2 of them being complicated by Ebstein's anomaly. Nineteen of 22 paroxysms were terminated by a rapid intravenous injection of ATP of 2 to $15 \mathrm{mg}$ with a mean dose of $0.78 \pm$ $0.48 \mathrm{mg} / \mathrm{kg}$ (SD). Termination of the tachyardia occurred at 7 to $17 \mathrm{sec}$ after the injection, with a mean value of $10.3 \pm 3.0 \mathrm{sec}$ and was caused by a blockade of the antegrade A-V conduction. Immediately after the termination of AVRT, transient sinus bradycardia was observed in all cases and A-V block developed in one. Two patients complained brief chest dicomfort. All of these lasted very short and did not require any treatment. These results suggest that ATP is a safe and effective drug for quick termination of AVRT in infants and children. Emphasis should be placed on the rapid bolus injection of the drug. Also, pretreatment with sedatives appeared to give higher success rate.

\section{$-206-$ \\ ELECTROPHYSIOLOGIC EFFECTS AND THERA- PEUTIC USE OF ADENOSINE AND ATP FOR TERMINATING PAROXYSMAL SUPRAVEN- TRICULAR TACHYCARDIA}

Akira Nozaki, Yoshihisa Kurachi, Iku Toda, Yuji Murakawa, Toshiaki Nakajima, Kiyoshi Kawakubo, Tsuneaki Sugimoto. 